patients to a different TNF inhibitor if they responded inadequately or developed adverse effects, although this approach is unsupported by evidence.

Medicare only covers infliximab, and managed-care organizations require patients to pay high copays or all costs of etanercept or adalimumab. Nonetheless, etanercept was the most popular choice, because of its favorable efficacy and fewer perceived side effects compared with adalimumab and infliximab.

Original article Kamal KM et al. (2006) Use of tumor necrosis factor inhibitors in rheumatoid arthritis: a national survey of practicing United States rheumatologists. Joint Bone Spine 73: 718-724

\section{Damage to the amygdala might cause neuropsychiatric symptoms in patients with SLE}

The blood-brain barrier normally prevents antibodies to the $\mathrm{N}$-methyl-D-aspartic acid receptor (NMDAR) from binding to and killing brain neurons, but the barrier can be damaged, for example by the stress hormone epinephrine. In rodent models of systemic lupus erythematosus (SLE), epinephrine-mediated damage to the blood-brain barrier results in antiNMDAR-mediated damage to the amygdala. Emmer and colleagues used MRI to investigate whether patients with SLE or neuropsychiatric SLE have structural changes in their brains, and whether such changes correlate with the presence of anti-NMDAR antibodies.

The authors enrolled 21 patients with SLE, 37 patients with neuropsychiatric SLE, and 12 sex-matched, age-matched control individuals. All patients underwent diffusion-weighted MRI to quantify structural brain damage, and the anti-NMDAR antibody status of a subgroup of eight patients with SLE was assessed (half of whom were anti-NMDAR ${ }^{+}$).

In patients with SLE or neuropsychiatric SLE, tissue integrity was decreased only in the amygdala (by a similar extent in both conditions) compared to that of controls. This damage was worse in anti-NMDAR ${ }^{+}$than in anti-NMDAR ${ }^{-}$patients.

In patients with SLE, anti-NMDAR antibodies seem to specifically target the amygdala. Selective damage might be caused by a breach of the blood-brain barrier at the amygdala only. The authors speculate that stress hormones increase the vulnerability of specific brain structures, by increasing the local permeability of the blood-brain barrier at those locations. They suggest that this pathogenetic mechanism might link severe stress and neuropsychiatric syndromes.

Original article Emmer BJ et al. (2006) Selective involvement of the amygdala in systemic lupus erythematosus. PLoS Med 3: e499

\section{Smoking increases the risk of cartilage loss in men with knee OA}

Animal and in vitro studies indicate that tobacco smoking impairs chondrocyte function (by inhibiting cell proliferation and extracellular matrix synthesis) and cartilage metabolism. Amin and colleagues, therefore, investigated the effects of smoking on cartilage loss and knee pain in men with knee osteoarthritis (OA).

Their 30 month study evaluated 159 men (mean age 68 years) with knee OA who participated in the Boston OA of the Knee Study (a natural history study). At baseline and after 15 and 30 months, patients' smoking status and knee pain were recorded, and they underwent $\mathrm{MRI}$ of the most symptomatic knee.

At baseline 19 patients were current smokers, 4 of whom quit during follow-up, and 1 nonsmoker started to smoke. After adjustment for potentially confounding variables, current smokers were more likely than current nonsmokers to have cartilage loss in the medial compartment of the tibiofemoral joint and the patellofemoral joint, and to report greater overall knee pain throughout the study. The mechanism for this association is unclear; pain is unlikely to be directly caused by cartilage loss, because cartilage does not contain pain fibers. Smoking might directly affect other articular structures, or modify the musculoskeletal pain threshold. The authors speculate that smoking-mediated oxidative stress or tissue hypoxia impairs cartilage metabolism.

The authors suggest that current smoking might exacerbate progression of symptomatic knee $\mathrm{OA}$, and that the effects of smoking might be greatest on cartilage that is already damaged.

Original article Amin S et al. (2007) Cigarette smoking and the risk for cartilage loss and knee pain in men with knee osteoarthritis. Ann Rheum Dis 66: 18-22 